\title{
Faults, Fractures and Karst Zones Characterization in a Pre-Salt Reservoir using Geometric Attributes.
}

*Tatiana A. S. Oliveira (Petrobras), Nathalia M. S. M. Cruz (Petrobras), José M. N. Cruz (Petrobras), Rejhane S. Cunha

(Petrobras), Marcílio Matos (SISMO).

\section{Copyright 2019, SBGf - Sociedade Brasileira de Geofísica}

This paper was prepared for presentation during the $16^{\text {th }}$ International Congress of the Brazilian Geophysical Society held in Rio de Janeiro, Brazil, 19-22 August 2019.

Contents of this paper were reviewed by the Technical Committee of the $16^{\text {th }}$ International Congress of the Brazilian Geophysical Society and do not necessarily represent any position of the SBGf, its officers or members. Electronic reproduction or storage of any part of this paper for commercial purposes without the written consent of the Brazilian Geophysical Society is prohibited.

\section{Abstract}

This study aims to present a work methodology with geometric attributes, focusing on faults, fractures and karst system characterization. The attributes were computed in the ASPII - SISMO platform and the methodology was applied in a pre-salt reservoir of Santos Basin. With this methodology application, the geometric attributes aided in the reservoir structural characterization, through the determination of areas with greater probability of fracturing, definition of the direction of fracture lineations and families, estimates of karst zones and sub-seismic faults.

\section{Introduction}

The reconnaissance of areas of higher fracture probability in seismic is based on the identification of the changes of amplitude, phase, frequency, dip, continuity and reflector configuration. Seismic attributes that provide quantitative measurements assist in the recognition of these areas when interpreted in conjunction with well profiles and production tests. The information from the analysis of these geometric attributes, together with the horizons and faults mapped and well fracture data, is used as basis for the structural framework building of the geological models of fractured reservoir. In this context, this study will present a methodology to work with geometric attributes focusing on the geological heterogeneities identification that are not easily detected in traditional amplitudes volumes (fractures, sub-seismic faults and karstification features). And present an application example in a pre-salt reservoir from Santos Basin.

\section{Method}

Geometric attributes widely used in geological discontinuities identification such as Semblance and Variance emphasize the regions of greater spatial amplitude variability and can indicate areas of greater fracturing probability or even, delineate reservoir limits and highlight vug/Karstification zones. Nowadays there are more sophisticated methods of calculating geometric attributes that take into account the three-dimensional sample space and estimate the vector of maximum correlation between the amplitude samples. As products are estimated Dip (structural dip) and Azimuth volumes that are used as input for calculations of curvature and coherence attributes (figure 1).

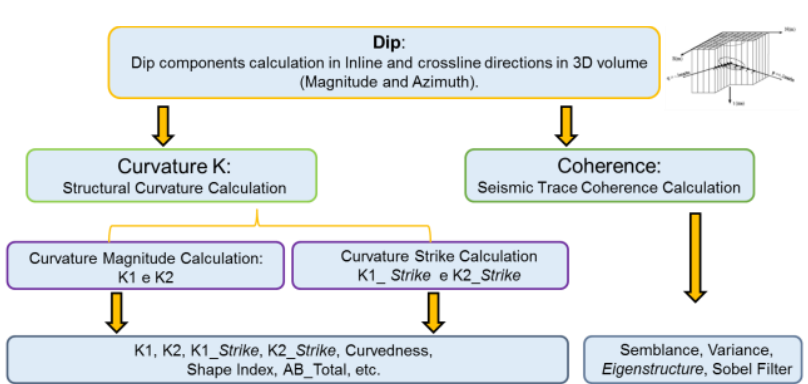

Figure 1: Flowchart for curvature and coherence attributes calculation estimated from Dip and Azimuth volumes (modified from Marfurt, 2007).

The coherence attributes use semblance algorithms (Semblance attribute) (Marfurt et al, 1998) and auto structure (Coherence Eigenstructure attribute) (Chopra and Marfurt, 2007), and take into account the lateral amplitude variation along the structural dip, and result in a small-scale discontinuity (high frequency) volume.

The algorithms calculate the coherent energy ratio of the seismic traces of a small volume around each point of the seismic volume and thus, if all traces are identical or very similar, the mean energy to the total energy ratio will have a unit value, or close to one respectively. On the other hand, if the seismic traces are not similar, such as those observed near geological faults, the energies ratio will have values close to zero.

Figures 2 and 3 illustrate schematically how the semblance and coherence eigenstructure algorithms are implemented.

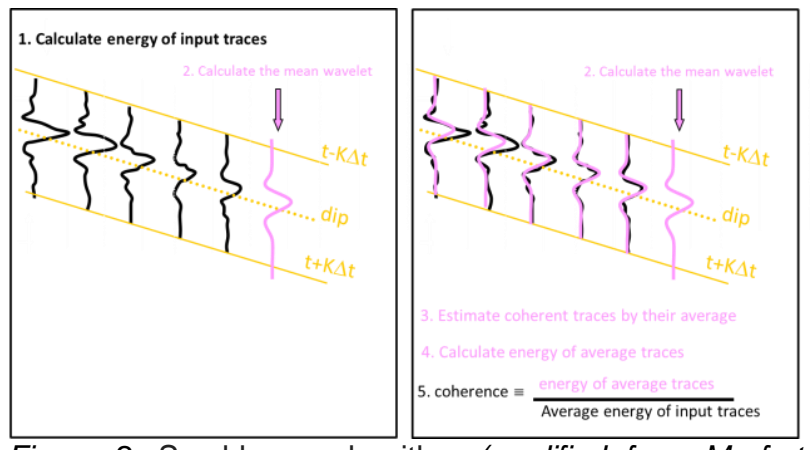

Figure 2: Semblance algorithm (modified from Marfurt, 2007). 


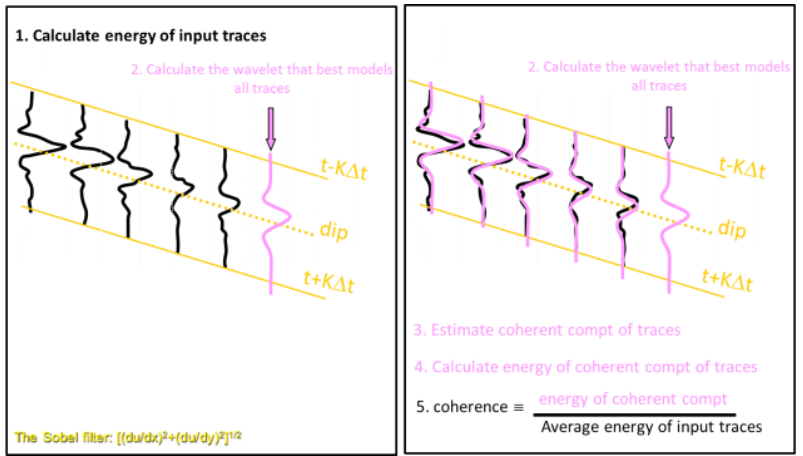

Figura 3: Auto structure algorithm (coherence eigenstructure) of energy ratio (modified from Marfurt, 2007).

The curvature attributes use algorithms that estimate the curvature $\mathrm{K}$ as the inverse of the radius of the circle tangent to a surface or reflector at each generic point of it, defined by the vectors tangent $\mathrm{T}$ and normal vector $\mathrm{N}$ (figure 4).

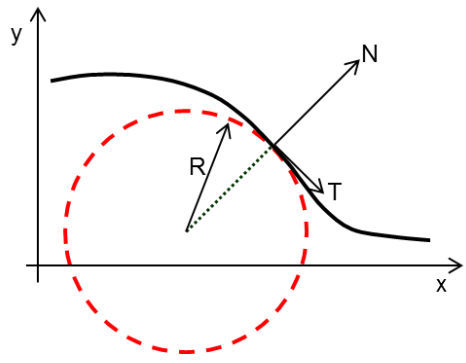

Figure 4: The curvature can be defined as the inverse of the radius of the osculating circle at each point of the curve (Marfut, 2007).

The algorithms calculate the most positive curvature, $\mathrm{K} 1$, and most negative curvature, $\mathrm{K} 2$, along the reflector and their combination makes it possible to describe the geometry and orientation of related structures, such as anticlines, synclines, mounds, and collapses (figure 5). From these geometric features it is possible to relate them to geological structures such as faults and fractures lineaments associated with the directions of the anticline and syncline axes and the mounds and collapses with Karstificated features.

Attributes related to $\mathrm{K} 1$ and $\mathrm{K} 2$ curvatures combination as Curvedness and Shape Index (Marfut, 2007) (figure 5), describe how curved the geometry is, independent of the concavity and provides an index for each geometric shape, which varies from -1 to +1 , respectively.

The methodology developed consisted of the integrated analysis of the curvature and coherence attributes.

In the curvature attributes, the K1 (most positive curvature) volume, and K2 (most negative curvature), the same color scale was used and in a symmetrical way. Therefore, it was possible to separate in the $\mathrm{K} 1$ volume, the geological structures with downwards concavity as the anticlines and mounds, in which the values of $\mathrm{K} 1$ are positive and isolate the collapses, in which $\mathrm{K} 1$ has negative values. The same process was applied in the K2 volume, in which the K2 negative values refer to the upwards concavity structures associated with the synclines and collapses, and the K2 positive values refer to the mounds (figure 5).

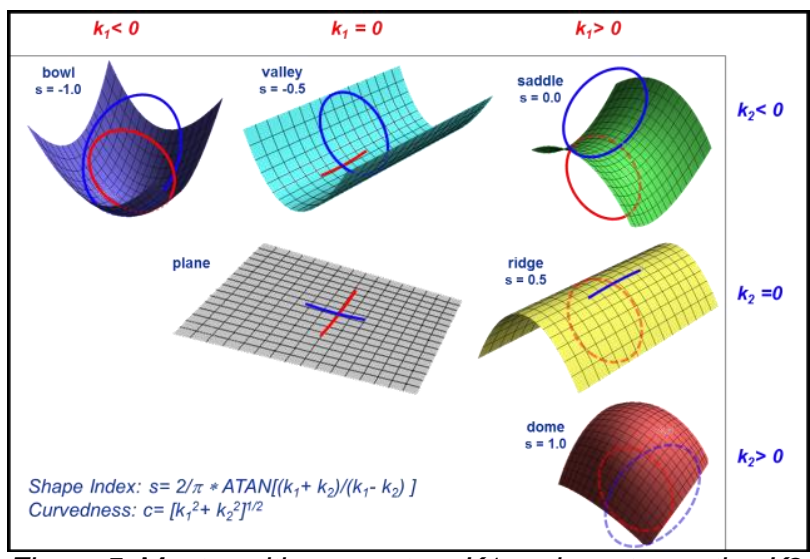

Figure 5: Most positive curvature K1 and most negative K2, and its association with $3 D$ geometric shapes (Marfut, 2007).

By isolating $\mathrm{K} 1$ positive and $\mathrm{K} 2$ negative values representing mainly anticlines and synclines, the axes directions of these structures were defined and the maps were colored in these directions. This attribute is called Strike of K1 and Strike of K2 (Marfut, 2007).

Combining the $\mathrm{K} 1$ and $\mathrm{K} 2$ volumes, the Curvedness and Shape Index attributes were calculated (figure 5). In Curvedness, which represents the curvature energy, the most positive values were isolated and this result was combined with the Shape Index attribute, that is, the more curved structures were colored by their corresponding geometry.

In the coherence attributes (Semblance and Eigenstructure) maps were generated and from the coherent energy calculation the Sobel Filter attribute was computed (Marfut 2007) (figure 3), known as the edge detection attribute.

The attributes maps generated along and in the intervals between the mapped horizons were compared with the interpreted faults and with the fractures data described in the image profiles from wells.

It's important to emphasize that the curvature and coherence attributes must be studied in an integrated way to provide more coherent interpretations of the geological structures.

\section{Results}

Figures 6 and 7 show the maps extracted along the horizon from the top of the reservoir in the $\mathrm{K} 1$ and $\mathrm{K} 2$ curvature volumes respectively.

Analyzing the maps of figures 6 and 7 on the left, it is possible to identify the areas with the highest probability of fracturing, that is, those in the region of the anticlines and synclines axes. And the collapses and mounds (figures 6 and 7 on the right) that occur mainly on the east edge of the reservoir. 


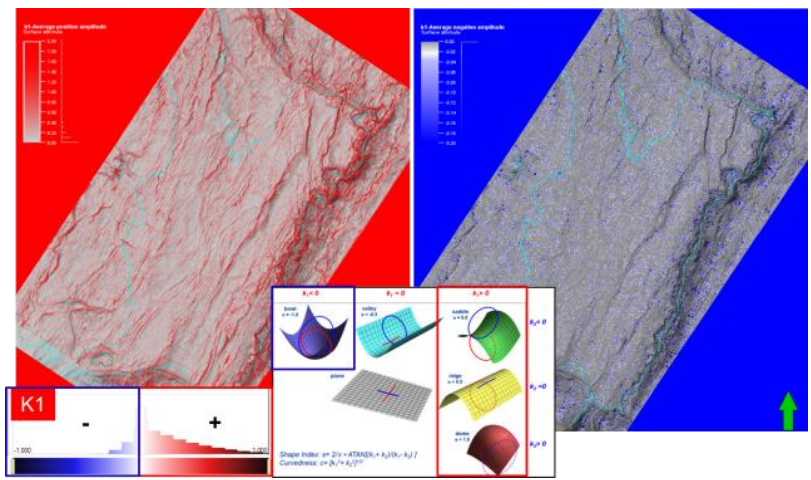

Figure 6: Maps extracted along the horizon from the top of the reservoir at $K 1$ curvature volume. To the left, in red, are isolated the positive values, that represent the anticlines and mounds. To the right, in blue, are the $K 1$ negative values that represent the collapses.

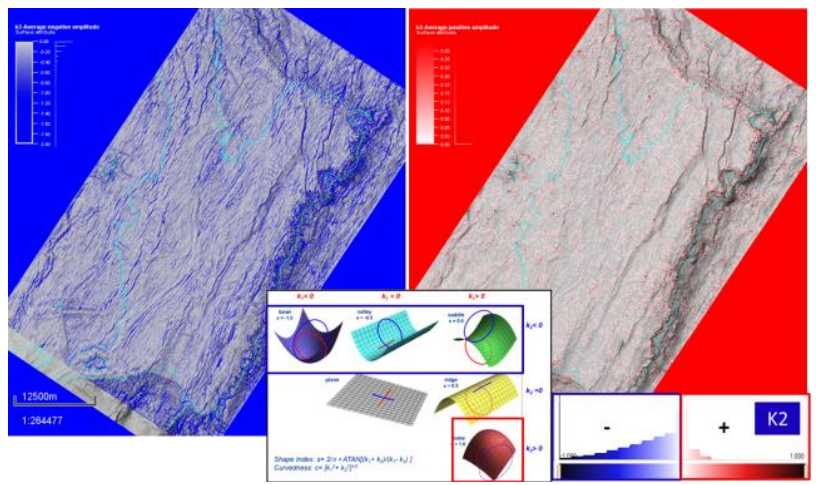

Figure 7: Maps extracted along the horizon from the top of the reservoir at K2 curvature volume. To the left, in blue, are isolated the negative values that represent the synclines and collapses and to the right, in red, the K2 positive values that represent the mounds.

K1 positive values were isolated and colored by the anticlinal axis direction (K1 Strike attribute) (figure 8). In the sequence the color scale was filtered in the main directions identified and compared with the faults mapped stereogram (figure 9).

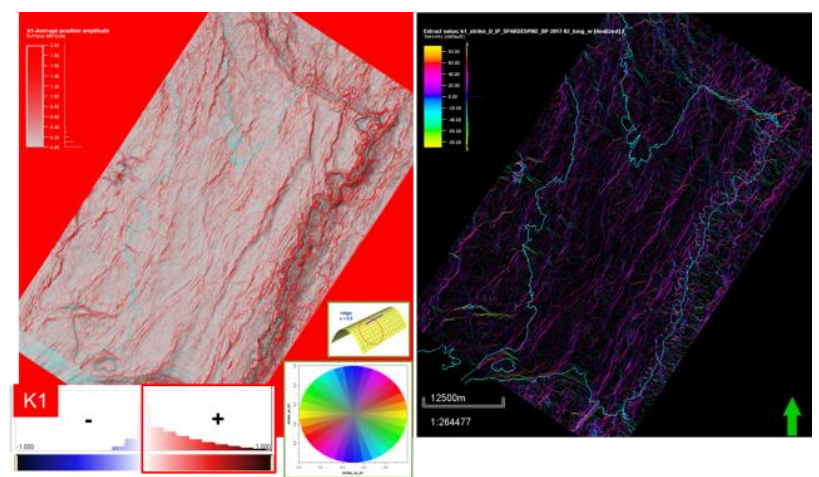

Figure 8: Maps extracted along the horizon from the top of the reservoir at $K 1$ Strike curvature volume. On the left, in red, are isolated the $K 1$ positive values, which represent the anticlines and mounds and the right, are the anticlines axes colored by the strike direction.

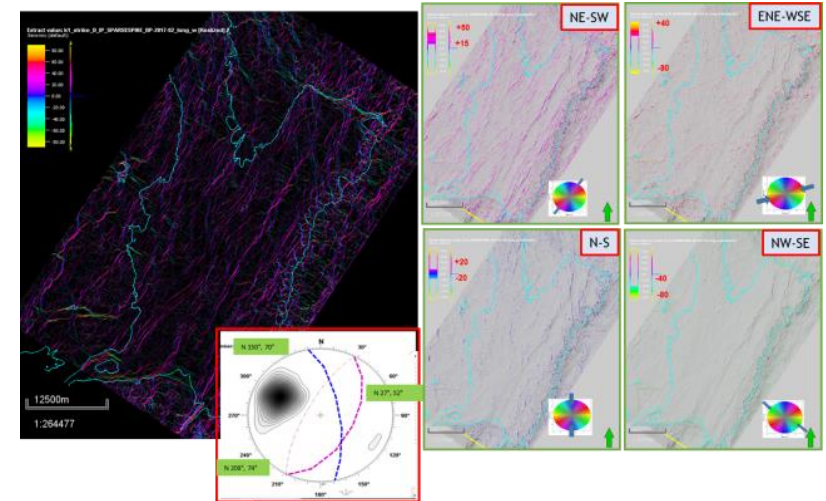

Figure 9: Maps extracted along the horizon from the top of the reservoir at K1 Strike curvature volume. On the left, the map shows the axes anticlines colored by strike direction and the faults mapped stereogram. To the right, the four maps show the four main discontinuities directions separately.

Examining the maps in the figures 8 and 9 it is noted that the main discontinuities directions are NE-SW and N-S, the same direction of the main mapped faults, which suggests that they may have been formed by the same tectonic event that originated the faults.

The strike direction fracture data interpreted in the well image profiles were plotted on the K1_Strike attribute map (figure 10). This analysis allowed identifying, from the wells data and comparing with the attribute map, four main discontinuity families: NE-SW, ENE-WSE, NS, NW-SE, coincident with the discontinuity's directions presented in figure 9.

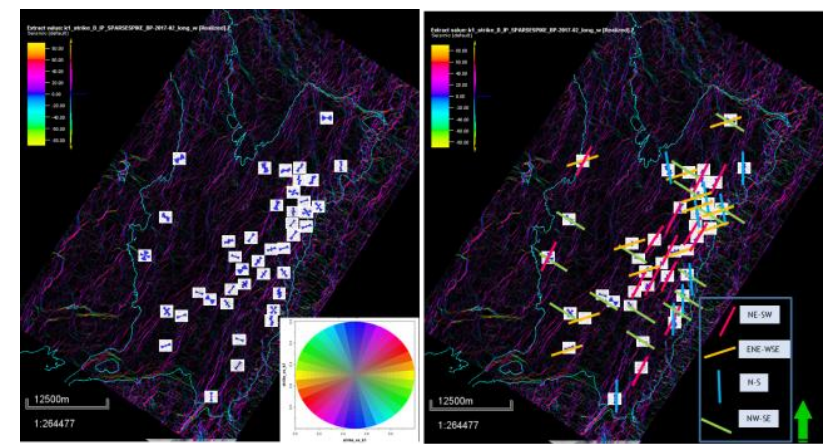

Figure 10: Map extracted along the horizon from the top of the reservoir in the K1_Strike attribute with the fractures strike direction stereogram from well image profile on the left. On the right the interpretation of the four main family's fractures NE-SW, ENE-WSE, NS and NW-SE.

The association of the $\mathrm{K} 1$ and $\mathrm{K} 2$ curvature volumes allowed the calculation of the Curvedness attribute and the combination of it with the Shape Index attribute as shown in figures 11 and 12. 


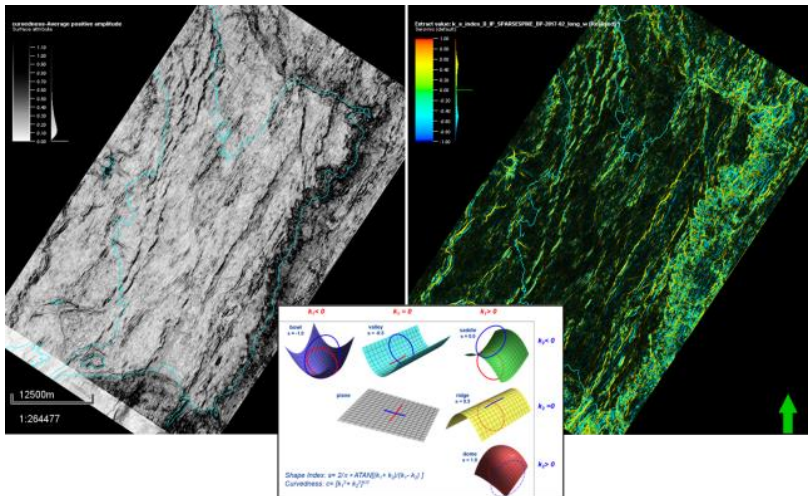

Figure 11: Maps extracted along the horizon from the top of reservoir at Curvedness volume on the left and the its combination with the Shape Index attribute.

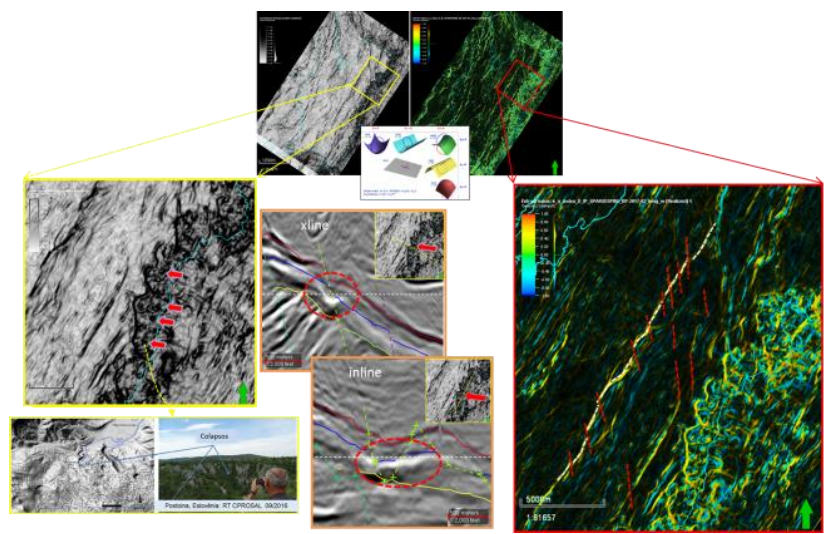

Figure 12: Zoom on the maps of figure 11, showing possible karst features to the left and lineages fractures to the right.

Analyzing the zoom details in attributes maps from figure 11 , shown in figure 12, to the left, it can be observed that, on the east border there are rounded features in map, which in the inline and crossline directions resemble karst collapses. Figure 12, on the right, shows discontinuities marked by white and red dotted lines that are suggested to be sub-seismic fracture lineaments.

Figure 13 shows the coherence attribute maps, Semblance on the left and the Eigenstructure on the right.
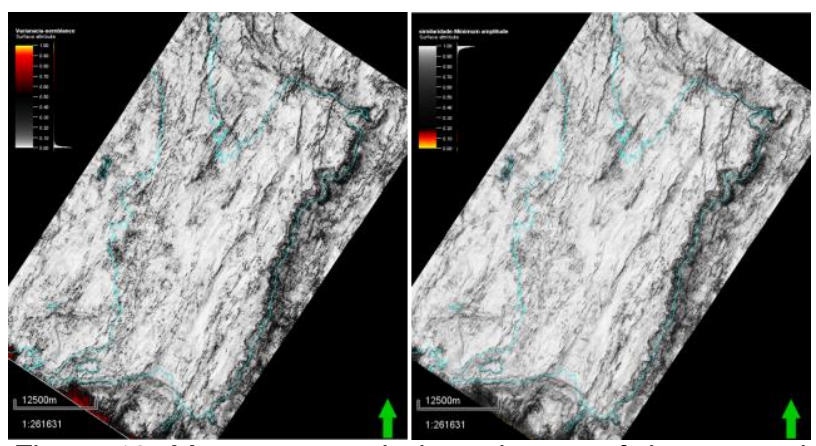

Figure 13: Maps extracted along the top of the reservoir horizon at Semblance volume on the left and Eigenstructure volume on the right.
The maps of figure 13 show that the main discontinuities are associated to the eastern edge of the reservoir, but with less continuity, as seen in the curvature attributes.

The maps generated from the Sobel Filter attribute volume are shown in Figures 14 and 15.
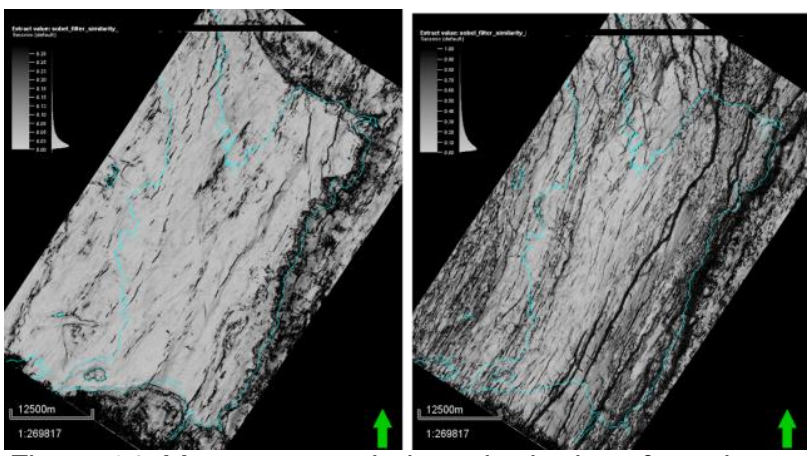

Figure 14: Maps extracted along the horizon from the top of the reservoir to the left and map from the top of the basement to the right in the Sobel filter attribute volume.

Observing the zoom on the maps of figure 14 shown in figure 15 , on the left, it is possible to visualize in map view and in the seismic section probable features of karst relief, associated with possible falls of blocks, as observed in the Jandaíra Formation, Potiguar Basin (Orildo et al). This feature can be visualized in the profiles of three wells drilled in the region that show "blocks", circled in blue, with bedding layers and a sudden change in the dip direction. To the right, on the top of basement map, it is possible to notice several discontinuities associated with the subseismic faults.
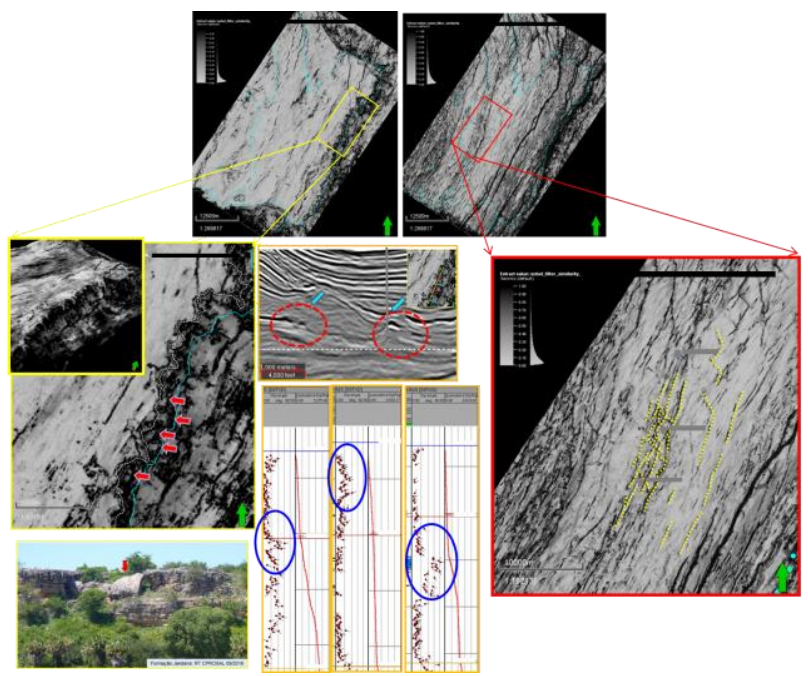

Figure 15: Zoom in the maps of figure 14, showing, on the left, possible karst features in map, seismic section and the three wells profiles. To the right, on the top of basement map, it is possible to notice several discontinuities associated with the sub-seismic faults. 


\section{Conclusions}

For this study, the good results obtained from the methodology application can be classified as satisfactory. Since with the use of geometric attributes interpretation providing a better structural characterization and identification of areas with greater probability of fracturing, sub-seismic faults fractures lineations and karst zones

Therefore, the attributes studied proved to be a high potential tool to support in the interpretation of the faults, fractures and karst system.

\section{Acknowledgments}

I would like to thanks Petrobras for the opportunity to develop this study, the geophysicist Alexandre Maul for the review and all the colleagues who contributed direct or indirectly.

\section{References}

Marfurt, K. J., R. L. Kirlin, S. L. Farmer, and M. S. Bahorich,1998, 3-D seismic attributes using a semblance based coherency algorithm: Geophysics, 63, 1150-1165.

Kirlin, R. L., and W. J. Done, 1999, Covariance analysis for seismic signal processing: Geophysical Developments Series, SEG, 355p.

Chopra, S., and K. J. Marfurt, 2007, Seismic attributes for prospect identification and reservoir characterization: SEG Geophysical Development Series, 11.

Orildo L. Silva, Francisco H.R. Bezerra, Rubson P.Maia, Caroline L. Cazarin, 2017, Karst landforms revealed at various scales using LiDAR and UAV in semi-arid Brazil: Consideration on karstification processes and methodological constraints: Geomorphology 295 (2017) 611-630. 